\title{
Mycoplasma Pneumoniae-Induced Rash and Mucositis: A Systematic Review of the Literature
}

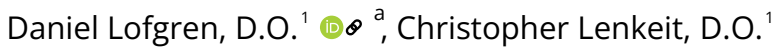 \\ ${ }^{1}$ Graduate Medical Education, Otolaryngology - Head \& Neck Surgery Resident, McLaren Oakland Hospital, Pontiac, MI, USA \\ Keywords: mycoplasma pneumoniae, mycoplasma pneumoniae-induced rash and mucositis, mucositis, rash, mirm, mim \\ https://doi.org/10.51894/001c.25284
}

\section{Spartan Medical Research Journal}

Vol. 6, Issue 2, 2021

\section{INTRODUCTION}

Mycoplasma pneumoniae (MP) is a common respiratory pathogen that can result in community-acquired pneumonia (CAP). Approximately $25 \%$ of patients diagnosed with MP experience extrapulmonary manifestations. Mycoplasma-induced rash and mucositis (MIRM) was coined as a unique disease process in 2014. MIRM has prominent mucositis with or without a characteristic vesiculobullous and/or atypical targetoid eruption. Appropriate identification of this disease is important because it has a milder disease course with low rates of sequelae, and lower mortality compared to Stevens-Johnson syndrome, erythema multiforme, and toxic epidermal necrolysis. The objective of this systematic review was to examine the English literature on Mycoplasma Pneumonia-induced rash and mucositis since the establishment of its diagnosis in 2014.

\section{METHODS}

The following online databases were used to identify appropriate studies that met the established inclusion and exclusion criteria: Pubmed, Cochrane, MedLine, Health Evidence, EPPI center, Allied Health Evidence. The following MesH search terms were used to further identify articles; "Mycoplasma pneumoniae induced rash and mucositis," "Mycoplasma pneumoniae rash and mucositis," "Mycoplasma pneumoniae rash," "Mycoplasma pneumoniae mucositis," "MIRM," "Mycoplasma induced rash and mucositis," "Mycoplasma rash and mucositis," "Mycoplasma rash," and "Mycoplasma mucositis." Data was extracted following the Preferred Reporting Items for Systematic Reviews and Meta-Analyses (PRISMA) guidelines.

\section{RESULTS}

One hundred and seventy-five records were initially screened, and nineteen studies were included in the review, leading to a total of 27 patients. Patients had a mean age of 16 years old (Range 4 - 46 years old), with the majority being males (74\%). Pulmonary symptoms tended to precede extrapulmonary symptoms on an average of 7.8 days. Extrapulmonary symptoms consisted of oral lesions (96.3\%) followed by ocular lesions (92.6\%) and genital lesions (59.3\%). Female patients were more likely to have genital lesions $(71.4 \%)$ when compared with male patients (55\%). Cutaneous rashes occurred in approximately one-half of the patients, which supports the theory that MIRM is a separate clinical entity from SJS and other related skin disorders.

Confirmatory testing for MIRM was performed using IgM/IgG Mycoplasma antibody testing or PCR in 19 (66.7\%) and 6 (22.2\%) patients respectively, although four cases reported the use of both serology and PCR, while five did not report confirmatory testing. Systemic antibiotics were used frequently in treatment 22 patients $(77.8 \%)$ and $27(100 \%)$ of the patients received various supportive care. Approximately 11 (37\%) patients of reported cases used systemic steroids to reduce systemic inflammation. Other systemic treatments were used in six (21.4\%) cases, and included intravenous immunoglobulins and cyclosporine A. Only eight patients (22.2\%) reported having any lasting sequelae. 


\section{CONCLUSION}

Mycoplasma-induced rash and mucositis is a recently described extra-pulmonary manifestation of Mycoplasma pneumoniae infections. To the best of the authors' knowledge, this is the first systematic review of the MIRM literature since the introduction of the diagnosis in 2014. The authors hope that this review can serve to better our current understanding and lead to improved identification, work-up, and treatment of this disease. One notable limitation of this study is the relatively small sample size, which is due to the recent introduction of the term.

\section{INTRODUCTION}

Mycoplasma pneumoniae (MP) is a common respiratory pathogen that can result in community-acquired pneumonia (CAP). ${ }^{1}$ One 2016 meta-analysis reported MP's prevalence as $10.1 \%$ of all CAP, with higher rates in children (17.6\%) compared to adults (7.2\%). ${ }^{1}$ Approximately $25 \%$ of patients diagnosed with MP experience extrapulmonary manifestations, which include pericarditis (i.e., inflammation of pericardium), thrombosis (i.e., blood clot), hepatitis (i.e., liver inflammation), hemolytic anemia (i.e., destruction of red blood cells), arthritis (i.e., inflammation of joints), encephalitis (i.e., inflammation of brain), glomerulonephritis (i.e., inflammation of kidneys), mucositis (i.e., mucosal inflammation), and varying dermatologic manifestations. $^{2-6}$ Historically, reported dermatologic manifestations of Mycoplasma pneumoniae were considered to be on the spectrum of erythema multiforme (EM), Steven-Johnson-Syndrome (SJS), and toxic epidermal necrolysis (TEN). ${ }^{3}$ EM is a skin immune rection appearing as raised red rashes in many different shapes, versus SJS which consists of a painful rash that blisters and sheds skin over body and mucous membranes. Toxic epidermal necrolysis is a more severe form of SJS, covering more surface area of the body. ${ }^{3}$ One smaller retrospective review of 30 pediatric patients looked for possible etiologies of EM and found that over $13.3 \%$ tested positive for MP. ${ }^{7}$

Although historically MP related mucocutaneous disease has fallen within the spectrum of EM, SIS, and TEN; recent literature has proposed that it be to be its own separate disease process. Canavan and colleagues performed the largest systematic review to date and were the first to coin the term Mycoplasma-induced rash and mucositis (MIRM) as a unique disease process in $2014 .^{2}$ This was based on their analysis of 202 cases of mucocutaneous (mucous membrane and skin) disease in patients that tested positive for $M y$ coplasma pneumoniae. Clinically, Canavan et al noted a varying degree of mucosal involvement with or without cutaneous involvement. They found a distinct disease morphology that did not fit into the established EM, SJS, and TEN diagnoses. MIRM has prominent mucositis (mucosal inflammation) with or without a characteristic skin vesicles and/or atypical target shaped eruption that one might see in the SIS spectrum. MIRM also generally has a milder disease course with low rates of sequelae, and lower mortality compared to EM, SJS, and TEN. Other studies have noted that the pathophysiology and treatment of this distinct clinical entity differs from previously described Mycoplasma induced erythema multiforme. ${ }^{2,4,7}$

\section{PATHOPHYSIOLOGY}

The pathophysiology of MIRM is still not fully understood and many theories have been proposed. The most widely accepted theory suggests cloning of B cells with cutaneous immune complex deposition and complement formation causes extrapulmonary symptoms. ${ }^{2,4}$ Molecular mimicry between mycoplasma's adhesion molecules and keratinocyte (i.e., skin cell) antigen has also been proposed but is less widely accepted. $2,4,5,8,9$ There is minimal information regarding histology of the mucocutaneous lesions that have been reported in the literature. Cutaneous leukocytoclastic vasculitis (i.e., inflammation of small capillary vessels) has been associated with Mycoplasma pneumonia in the past and is characterized by neutrophilic perivascular infiltrate around the lesional sites. ${ }^{3}$ Amode et al. described a Toxic Epidermal Necrolysis-like histologic pattern consisting of minimal dermal change with intense and keratinocyte apoptosis in 14 patients with MIRM. ${ }^{10}$

\section{EPIDEMIOLOGY AND SYMPTOMATOLOGY}

Generally, patients suffering from MIRM are afflicted in the winter months, are male (60-66\%), young (8.7 to 11.9 years old), and experience prodromal (i.e., non-specific) symptoms including fever, malaise, and cough on average 7-10 days before mucocutaneous symptoms..$^{2,4,8,10}$ In the original description, Canavan and colleagues noted sparse cutaneous involvement - defined as a few scattered lesions in $47 \%$ of patients, compared with severe mucositis alone (34\%) and moderate cutaneous involvement alone (19\%). Cutaneous lesions of the extremities (47\%) were more common than lesions of the trunk (23\%) and generalized involvement (31\%). ${ }^{2,10}$ Reported cutaneous lesions varied in appearance from vesiculobullous (77\%) to targetoid (48\%), papular (14\%), macular (12\%), and morbilliform (9\%). ${ }^{2,4,5}$ One prospective cohort study of 152 children with CAP by Sauteur et al, revealed 44 patients (28.9\%) tested positive for MP, and of these children, ten (22.7\%) developed mucocutaneous lesions. Of these ten patients, five developed maculopapular skin eruptions (11.4\%), two had urticaria (4.5\%), and three patients had mucocutaneous disease $(6.8 \%) .^{8}$

The involvement of mucosal surfaces appears to be the hallmark associated with this disease. The oral cavity was involved in $94 \%$ of patients with symptoms ranging from erosions and ulcers to denuded tissue. ${ }^{2,4,5}$ Ocular involvement was the second most common extrapulmonary symptom occurring in $82 \%$ of patients. ${ }^{2,4}$ Patients presented with purulent bilateral conjunctivitis, photophobia, pseudomembrane formation, ulceration, and eyelid 
edema. ${ }^{2,4,5}$ Urogenital lesions occurred in approximately $63 \%$ of patients. Interestingly, in Canavan's study, only four of the 202 patients did not have mucosal involvement. $2,4,5,11$

\section{DIAGNOSIS}

Diagnosis of MIRM has historically been based on positive identification of Mycoplasma pneumoniae on clinical, radiological, and laboratory findings with associated extrapulmonary symptoms. Generally, patients are admitted to the hospital for symptoms of pneumonia and work up confirms the diagnosis of MP. Diagnosis is generally made with serologic testing using cold agglutinins, bullae cultures, and polymerase chain reaction (PCR), but more recently, some authors report using enzyme-linked immunoassays (ELISA) and Mycoplasma pneumoniae IgM antibody levels. ${ }^{2,9}$ Laboratory findings can include elevated c-reactive protein (CRP), erythrocyte sedimentation rate (ESR), and leukocytosis with left shift. $4,5,8,9,12,13$ Other pathogenic causes of mucocutaneous lesions must be ruled out including Herpes Simplex Virus, Cytomegalovirus, Varicella-Zoster, Chlamydia species, Coxsackie virus, influenza B, Staphylococcus aureus, and a host of other autoimmune diseases. $4,7,13,14$

The proposed diagnostic criteria for classic MIRM includes clinical and laboratory evidence of atypical pneumonia caused by Mycoplasma pneumoniae with the following: $\geqslant$ 2 involved mucosal sites, less than $10 \%$ involved cutaneous surface area, few vesiculobullous lesions or atypical scattered targets with or without targetoid lesions. There are two proposed variants of MIRM called severe MIRM with extensive involvement of atypical targetoid lesions or blisters and MIRM sine (without) rash, which showed minimal morbilliform lesions with few vesicles. ${ }^{2,4,14}$ Interestingly, patients presenting with MIRM sine rash had higher rates of mucosal involvement: oral (100\%), ocular (92\%), and urogenital (78\%). ${ }^{2,4}$

Since the establishment of the MIRM classification system in 2014, there have been no comprehensive reviews of reported MIRM studies in the literature. The authors wish to systematically review the current literature to provide an up-to-date and comprehensive picture of the presentation, diagnosis, work-up, and treatment of this relatively uncommon disease.

\section{METHODS}

This study was designed as a systematic review of the literature of MIRM since the establishment of the diagnosis in 2014. The article selection process used can be seen in Figure 1, which follows the Preferred Reporting Items for Systematic Reviews and Meta-Analyses (PRISMA) guidelines. Inclusion criteria included any study type from January 2014 to April 2020 containing adult and/or pediatric patients who were clinically diagnosed with MIRM. English language or English translated papers were included, and the authors were required to have full access to abstract and manuscript. Exclusion criteria involved studies that were determined by the authors to be letters to the editor or opinion pieces. The following online databases were used

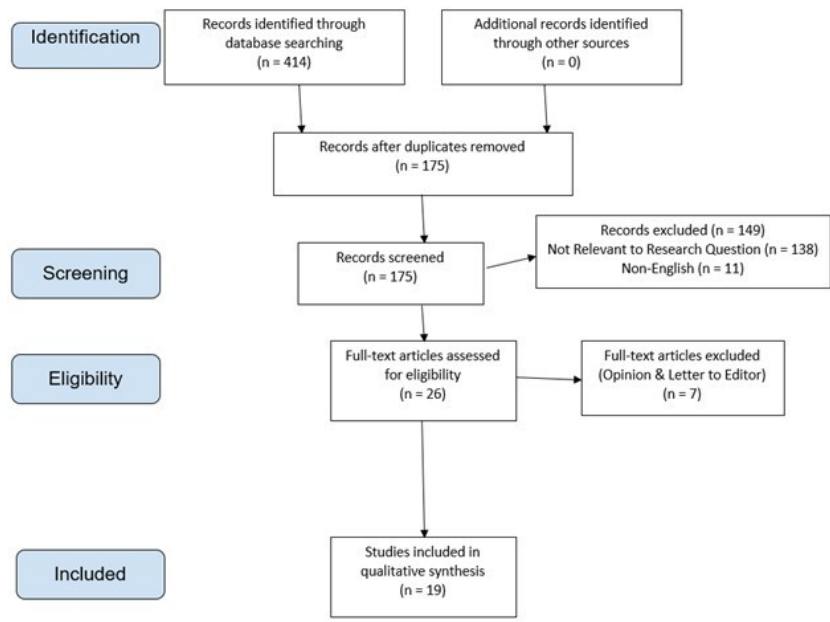

Figure 1. Article Inclusion directed by Preferred Reporting Items for Systematic Reviews and MetaAnalyses (PRISMA) guidelines.

to identify appropriate studies that met our inclusion and exclusion criteria: Pubmed, Cochrane, MedLine, Health Evidence, EPPI center, Allied Health Evidence. The following MesH search terms were used to further identify articles "Mycoplasma pneumoniae induced rash and mucositis," "Mycoplasma pneumoniae rash and mucositis," "Mycoplasma pneumoniae rash," "Mycoplasma pneumoniae mucositis," "MIRM," "Mycoplasma induced rash and mucositis," "Mycoplasma rash and mucositis," "Mycoplasma rash," and "Mycoplasma mucositis."

All abstracts for the studies were screened and reviewed by the lead authors (DL, CL) to determine if the studies met the inclusion or exclusion criteria above. For data collection, all studies were reviewed independently by all authors and added to a common Microsoft excel sheet with title and author and abstract. Duplicates were then removed after all databases were searched thoroughly using the terms above. The author team independently reviewed each paper to determine the significance of the topic and make sure that they met the current established criteria for MIRM. All authors followed the above criteria. Statistical results were reported in both numbers and percentages.

\section{RESULTS}

A thorough review of the literature from January 2014 to April 2020 revealed 19 studies with a total of 27 patients who met clinical criteria for MIRM since it was clarified diagnostically in 2014. All studies meeting the above inclusion criteria were case reports. Biological gender, age, pulmonary symptoms, extrapulmonary symptoms, diagnostic testing, results of chest imaging, treatments, and outcomes were recorded in Table 1. Patients ranged in age from 4 to 46 years of age with a mean age of 16 . Of the total patient population, 20 (74\%) patients were males. Symptoms, diagnosis, treatment, and sequelae can be seen in Table 2. Pulmonary symptoms varied from mild dyspnea and cough to severe respiratory distress.

The results of imaging also varied which is expected 
Table 1. Age and gender demographics for the patients included in this study

\begin{tabular}{|l|l|}
\hline Category & Number of Patients (Percentage/SD) \\
\hline Total Patients & 27 \\
\hline Male Patients & $20(74.1 \%)$ \\
\hline Female Patients & $7(25.9 \%)$ \\
\hline Mean Age (Years Old) & $16.3( \pm 9.95)$ \\
\hline Mode Age (Years Old) & 16 \\
\hline Median Age (Years Old) & 14 \\
\hline Range (Years Old) & $4-46$ \\
\hline
\end{tabular}

given MP's designation as an atypical pneumonia. Pulmonary symptoms tended to precede extrapulmonary symptoms by an average of 7.8 days. The most common extrapulmonary symptoms were oral lesions in 26 (96.3\%) patients followed by 25 patients with ocular lesions (92.6\%) and $16(59.3 \%)$ with genital lesions. Interestingly, female patients were more likely to have genital lesions, 5 (71.4\%), when compared with male patients, 11 (55\%). Cutaneous rashes occurred in approximately half of the patients, which supports that MIRM appears to be a separate clinical entity from SJS and other related skin disorders. Diagnosis of $M y-$ coplasma pneumoniae was done either clinically or with chest radiography in all cases. Confirmatory testing was performed using IgM/IgG Mycoplasma antibody testing or PCR in 19 (66.7\%) and 6 (22.2\%) patients respectively, although 4 cases reported the use of both serology and PCR, and 5 did not report confirmatory testing.

Twenty-two patients were given systemic antibiotics for treatment (77.8\%) and 27 (100\%) of the patients received supportive care, which included intravenous hydration, pain control, and topical medications for localized wounds (steroids or antibiotics). Approximately 11 (37\%) reported cases used systemic steroids to reduce systemic inflammation. Other systemic treatments included either intravenous immunoglobulins and cyclosporine A in 6 (21.4\%) cases. Only eight patients (22.2\%) reported having any lasting sequelae. Two patients noted hypo/hyperpigmentation changes, two patients noted ocular scarring and xerophthalmia, one patient had persistent cutaneous pain, another patient had chronic phimosis, and the final two patients noted residual and recurrent skin lesions.

\section{DISCUSSION}

Although there are no standardized treatment guidelines for patients suffering from MIRM supportive management is the mainstay of treatment, which includes pain management, intravenous hydration, and mucosal care. ${ }^{2,4,8,9,15}$ Overall, our study data reports that twenty-two (77\%) patients were treated with antibiotics, 11 (37\%) with corti- costeroids, and $11 \%$ with IVIG. No high-powered studies have compared resolution times or efficacy of treatments for MIRM. Recently, a case series of three patients with MIRM treated with cyclosporine A (CsA) reported a significantly shortened duration of hospital admission and morbidity if given within 48 hours of mucocutaneous eruption. Their patients stayed in the hospital for 5-7 days compared to the previously reported length with supportive care of 11-14 days. This appeared to translate into cheaper costs and less infectious disease risk when compared with IVIG alone. $^{14}$

Some patients may require increased levels of care including intensive care unit or burn center management. ${ }^{2,4}$ Sauteur and colleagues noted a statistically significant increase in hospital stay length in patients suffering from MIRM compared with non-mycoplasma EM patients (9.5 vs. 5.1 days) with a reported odds ratio (OR) of 9 (95\% CI, 1.4-81.4; $\mathrm{P}=0.01)$. They also reported increased oxygen requirements in patients with MIRM vs. CAP alone $(\mathrm{OR}=17.6$; $95 \% \mathrm{CI}, 1.5-984.1 ; \mathrm{P}=.007) .{ }^{8}$ The recurrence rate of MIRM has been reported as $8 \%$, while the mortality rate has been noted as 3\%, with all reported deaths prior to $1940 .^{2}$

Although the majority of MIRM patients are known to generally make a full recovery (81\%), a variety of complications have been noted in the literature. ${ }^{2}$ Compared with CAP, patients suffering from MIRM are more likely to develop long term sequelae. ${ }^{8}$ Orbital complications are noted in approximately $9 \%$ of patients and include conjunctival shrinkage, corneal ulceration, blindness, ocular synechiae, lash loss, and xerophthalmia (i.e., dry eyes). Postinflammatory pigmentation changes are noted in about $5.6 \%$ of cases. ${ }^{2,8}$ Oral and genital synechiae each occur in approximately $1 \%$ of patients. Other reported rare complications include genital adhesions, hematemesis (i.e., bloody emesis), epiglottitis (i.e., inflammation of the epiglottis), subcorneal pustulosis (i.e., pustules of the eye), B-cell lymphopenia (i.e., low B-Cell count), and death. $2,4,5,10$ Genetic susceptibility has also been theorized to play a role due to the reported $8 \%$ recurrence rate and distribution in families as reported in the literature. ${ }^{2,6}$ 
Table 2. Symptoms, diagnosis, and treatment of patients suffering from MIRM

\begin{tabular}{|c|c|c|c|c|c|c|c|}
\hline Category & Oral Symptoms & Ocular Symptoms & $\begin{array}{l}\text { Genital Lesions - } \\
\text { All Genders }\end{array}$ & $\begin{array}{l}\text { Genital Lesions - } \\
\text { Male Patients }\end{array}$ & $\begin{array}{l}\text { Genital Lesions - } \\
\text { Female Patients }\end{array}$ & Cutaneous Rash & \\
\hline Number of Patients & 26 & 25 & 16 & 11 & 5 & 15 & \\
\hline \multirow[t]{2}{*}{ Percentage (\%) } & 96.3 & 92.6 & 59.3 & 55.0 & 71.4 & 55.6 & \\
\hline & $\begin{array}{l}\text { Seropositivity } \\
\text { (IgM/lgG) }\end{array}$ & $\begin{array}{l}\text { Polymerase Chain } \\
\text { Reaction (PCR) }\end{array}$ & $\begin{array}{l}\text { Systemic } \\
\text { Antibiotics (PO/IV) }\end{array}$ & $\begin{array}{l}\text { Systemic Steroids } \\
\text { (PO/IV) }\end{array}$ & $\begin{array}{l}\text { Intravenous } \\
\text { Immunoglobulins } \\
\text { (IVIG) }\end{array}$ & Cyclosporine & Sequelae \\
\hline Number of Patients & 19 & 6 & 22 & 11 & 3 & 3 & 6 \\
\hline Percentage (\%) & 66.7 & 22.2 & 77.8 & 37.0 & 10.7 & 10.7 & 22.2 \\
\hline
\end{tabular}


The authors recognize that this study has inherent limitations. Due to the recent establishment of the diagnosis and the similar symptomology and mimicry to other skin disorders like TEN, SIS, and EM, MIRM has a limited number of published cases. This study provides the only comprehensive review of all of the cases reported in the literature since inception of the diagnosis, which will allow for further studies to compare their data. Another limitation of this study was the use of English only or English translated literature only. Unfortunately, all manuscripts included in the literature were either case reports or case studies and have inherently no power, so it's hard to draw high powered statistical evidence from them. Additionally, since our data is largely drawn from case studies there is some missing information regarding patients, including diagnostic testing, further description of supportive treatment, and cutaneous rash onset. This makes it difficult to standardize possible diagnostic and treatment protocols.

\section{CONCLUSION}

Mycoplasma-induced rash and mucositis is one of the more recently discovered extrapulmonary manifestations of $M y$ coplasma pneumoniae infections. The study is the first systematic review of the MIRM literature since the introduc- tion of the diagnosis in 2014. The authors hope that this review can serve to better our current understanding of this disease and lead to improved identification, work-up, and treatment of this disease.

\section{DISCLOSURE/COI}

The authors declare that they have no conflicts of interest to disclose

\section{FUNDING}

No funding was provided to the authors for the creation of this manuscript

\section{ACKNOWLEDGMENT}

The authors would like to thank both Dr. Olga Santiago Rivera, $\mathrm{PhD}$, and Dr. Carlos Rios-Bedoya ScD/MPH, for their guidance during the writing of this manuscript. Their assistance was invaluable in the design, production, and editing of this paper.

Submitted: January 16, 2021 EDT, Accepted: June 28, 2021 EDT 


\section{REFERENCES}

1. Marchello C, Dale AP, Thai TN, Han DS, Ebell MH. Prevalence of atypical pathogens in patients with cough and community-acquired pneumonia: a metaanalysis. Ann Fam Med. 2016;14(6):552-566. doi:10.13 70/afm.1993

2. Canavan TN, Mathes EF, Frieden I. Mycoplasma pneumoniae-induced rash and mucositis as a syndrome distinct from stevens-johnson syndrome and erythema multiforme: a systematic review. J Am Acad Dermatol. 2015;72(2). doi:10.1016/j.jaad.2014.0 $\underline{6.026}$

3. Narita M. Classification of extrapulmonary manifestations due to mycoplasma pneumoniae infection on the basis of possible pathogenesis. Front Microbiol. 2016;7. doi:10.3389/fmicb.2016.00023

4. Frantz GF, McAninch SA. Mycoplasma mucositis. In: StatPearls. Treasure Island (FL): StatPearls Publishing; 2019. https://www.ncbi.nlm.nih.gov/book s/NBK525960/. Accessed January 2020.

5. Martínez-Pérez M, Imbernón-Moya A, LobatoBerezo A, Churruca-Grijelmo M. Mycoplasma pneumoniae-Induced mucocutaneous rash: a new syndrome distinct from erythema multiforme? Report of a new case and review of the literature. Actas Dermosifiliogr. 2016;107(7):e47-e51. doi:10.1016/j.ade ngl.2016.06.005

6. Song H, Huang JT, Tan JK. Mycoplasma-induced rash and mucositis in a father and son. J Pediatric Infect Disease. 2018;37(7). doi:10.1097/inf.000000000 $\underline{0001881}$

7. Siedner-Weintraub Y, Gross I, David A, Reif S, Molho-Pessach V. Paediatric erythema multiforme: epidemiological, clinical and laboratory characteristics. Acta Dermato Venereologica. 2017;97(4):489-492. doi:10.2340/00015555-2569
8. Sauteur PMM, Theiler M, Buettcher M, Seiler M, Weibel L, Berger C. Frequency and clinical presentation of mucocutaneous disease due to mycoplasma pneumoniae infection in children with community-acquired pneumonia. JAMA Dermatol. 2020;156(2):144. doi:10.1001/jamadermatol.2019.360 2

9. Zão I, Ribeiro F, Rocha V, Neto P, Matias C, Jesus G. Mycoplasma pneumoniae-associated mucositis: a recently described entity. Eur J Case Rep Intern Med. 2018;5(11):1. doi:10.12890/2018_000977

10. Amode R, Ingen-Housz-Oro S, Ortonne N, et al. Clinical and histologic features of mycoplasma pneumoniae -related erythema multiforme: a singlecenter series of 33 cases compared with 100 cases induced by other causes. J Am Acad Dermatol. 2018;79(1):110-117. doi:10.1016/i.jaad.2018.03.013

11. Shah SS. Mycoplasma pneumoniae as a cause of community-acquired pneumonia in children. Clin Infect Dis. 2018. doi:10.1093/cid/ciy421

12. Kheiri B, Alhesan NA, Madala S, Assasa O, Shen M, Dawood T. Mycoplasma pneumoniae-associated fuchs syndrome. Clin Case Rep. 2017;6(2):434-435. do $\mathrm{i}: 10.1002 / \operatorname{ccr} 3.1350$

13. Goyal A, Hook K. Two pediatric cases of influenza $\mathrm{b}$ - induced rash and mucositis: stevens - johnson syndrome or expansion of the mycoplasma pneumoniae - induced rash with mucositis (MIRM) spectrum? Pediatr Dermatol. 2019;36(6):929-931. do i:10.1111/pde.13921

14. Li HO-Y, Colantonio S, Ramien ML. Treatment of mycoplasma pneumoniae-induced rash and mucositis with cyclosporine. J Cutan Med Surg. 2019;23(6):608-612. doi:10.1177/1203475419874444

15. Curtiss P, Melnick L, Sicco KL, Liebman TN. Mycoplasma pneumoniae, more than a lung disease. Dermatology Online Journal. 2018;24(6):13030/ qt8w993185. doi:10.5070/d3246040695 